\title{
Mechanism Design for Subadditive Agents via an Ex Ante Relaxation
}

\author{
SHUCHI CHAWLA, University of Wisconsin-Madison \\ J. BENJAMIN MILLER, University of Wisconsin-Madison
}

\begin{abstract}
We consider the problem of maximizing revenue for a monopolist offering multiple items to multiple heterogeneous buyers. We develop a simple mechanism that obtains a constant factor approximation under the assumption that the buyers' values are additive subject to a matroid feasibility constraint and independent across items. Importantly, different buyers in our setting can have different constraints on the sets of items they desire. Our mechanism is a sequential variant of two-part tariffs. Prior to our work, simple approximation mechanisms for such multi-buyer problems were known only for the special cases of all unit-demand or all additive value buyers.

Our work expands upon and unifies long lines of work on unit-demand settings and additive settings. We employ the ex ante relaxation approach developed by Alaei [2011] for reducing a multiple-buyer mechanism design problem with an ex post supply constraint into single-buyer problems with ex ante supply constraints. Solving the single-agent problems requires us to significantly extend a decomposition technique developed in the context of additive values by $\mathrm{Li}$ and Yao [2013] and its extension to subadditive values by Rubinstein and Weinberg [2015].
\end{abstract}

\section{INTRODUCTION}

Multi-parameter optimal mechanism design is challenging from both a computational and a conceptual viewpoint, even when it involves only a single buyer. Multi-parameter type spaces can be exponentially large, and multi-dimensional incentive constraints lack the nice structure of single-dimensional constraints that permits simplification of the optimization problem. As a result, optimal mechanisms can possess undesirable properties such as requiring randomness [Briest et al. 2010; Hart and Nisan 2012, 2013], displaying non-monotonicity of revenue in values [Reny and Hart 2015; Rubinstein and Weinberg 2015], and are in many cases computationally hard to find (see, e.g., Daskalakis et al. 2012, 2014). The situation exacerbates in multi-agent settings. Hartline [2016, chap. 8] identifies two further difficulties: multi-parameter agents impose multi-dimensional externality on each other that may not be possible to capture succinctly; and multi-parameter problems are typically not revenue linear, meaning that the optimal revenue does not scale linearly with the probability of service. Designing simple near-optimal mechanisms in such settings is a primary goal of algorithmic mechanism design.

In this paper we study the problem facing a monopolist with many items and many buyers, where each buyer is interested in buying one of many different subsets of items, and his value for each such subset is additive over the items in that subset. What selling mechanism should the monopolist use in such a setting to maximize his revenue? One challenge for the seller is that buyers may have heterogeneous prefer-

This work is supported by the National Science Foundation, grant CCF-1101429 and grant CCF-1320854. Authors' addresses: S. Chawla and J. B. Miller, Department of Computer Sciences, University of WisconsinMadison; email: \{shuchi,bmiller\}@cs.wisc.edu

Permission to make digital or hard copies of all or part of this work for personal or classroom use is granted without fee provided that copies are not made or distributed for profit or commercial advantage and that copies bear this notice and the full citation on the first page. Copyrights for components of this work owned by others than ACM must be honored. Abstracting with credit is permitted. To copy otherwise, or republish, to post on servers or to redistribute to lists, requires prior specific permission and/or a fee. Request permissions from permissions@acm.org.

EC'16, July 24-28, 2016, Maastricht, The Netherlands.

ACM 978-1-4503-3936-0/16/07 ...\$15.00.

Copyright is held by the owner/author(s). Publication rights licensed to ACM.

http://dx.doi.org/10.1145/2940716.2940756 
ences: some buyers are interested in buying a few specific items, others are indifferent between multiple items, and yet others have a long shopping list. We design the first approximation mechanism for this problem; our main result is a constant-factor approximation when buyers' values are additive up to a matroid feasibility constraint.

Our approximation mechanism has a particularly simple and appealing format - a sequential extension of standard two-part tariff mechanisms. Two-part tariffs for a single agent have the following structure. The buyer first pays a fixed entry fee and is then allowed to purchase any set of items at fixed per-item prices. The buyer may choose not to participate in the mechanism, in which case he does not pay the entry fee and does not receive any item. In our context, buyers pay (different) entry fees at the beginning, and then take turns (in an arbitrary but fixed order) to buy a subset of items at predetermined item-specific prices, subject to availability. There are many real-world examples of two-part tariffs, such as amusement park pricing; memberships for discount shopping clubs like Costco, Sam's Club, and Amazon's Prime; telephone services; and membership programs for cooperatives and CSAs. These mechanisms have long been studied in economics for their ability to effectively price discriminate among different buyers despite their relative simplicity. Armstrong [1999] shows, for example, that for an additive value buyer with independent item values and sufficiently many items, two-part tariffs extract nearly the entire social surplus.

Our work combines and significantly extends techniques from several different lines of work in mechanism design. We use the ex ante relaxation of Alaei [2011] to break up the multi-agent revenue maximization problem into its single-agent counterparts and capture the externalities among buyers through ex ante supply constraints. We solve the single-agent problems with ex ante supply constraints by adapting and extending the so-called core-tail decomposition technique of Li and Yao [2013], as well employing the prophet inequalities of Chawla et al. [2010a] and Feldman et al. [2016]. Finally, we use ideas from [Chawla et al. 2010a] to combine the resuting single-agent mechanisms sequentially and obtain a multi-agent approximation mechanism that is ex post supply feasible. While our main result applies to buyers with values additive up to a matroid constraint, parts of our approach extend to more general value functions such as those satisfying the gross substitutes condition.

\subsection{Multi-Parameter Mechanism Design: Previous Work}

This paper belongs to a long line of research on finding simple and approximately optimal mechanisms for multi-parameter settings under various assumptions on the buyers' value functions and type distributions, and on the seller's supply constraint. The first breakthrough along these lines was made by Chawla et al. [2007] who showed that the revenue of an optimal mechanism for a single unit-demand buyer can be approximated within a factor of 3 by an item pricing ${ }^{1}$, a mechanism that allows the buyer to choose any item to buy at fixed per-item prices. More recently, Babaioff et al. [2014] developed a similar result for a single buyer with additive values. ${ }^{2}$ They showed that the revenue of an optimal mechanism in this case is approximated within a factor of 6 by one of two simple mechanisms: an item pricing that fixes a price for each item and allows the buyer to choose any subset of items to buy, and a bundle pricing that allows the buyer to buy the grand bundle of all items at a fixed price. Observe that item pricing and bundle pricing are both two-part tariffs (with the entry fee or the per-item prices being zero, respectively).

Unit-demand and additive types are two extremes within a broader class of value functions that we call constrained additive values. A constrained additive buyer has a

\footnotetext{
${ }^{1}$ Chawla et al. [2010a] later improved this approximation factor to 2.

${ }^{2}$ This is the culmination of a series of papers including [Hart and Nisan 2012, 2013; Li and Yao 2013].
} 
value (drawn independently) for each item under sale; he is interested in buying a set of items that satisfies a certain downward-closed constraint; his value is additive over any such set. We have only recently begun to understand optimal mechanism design for a single agent with constrained additive values. Rubinstein and Weinberg [2015] proved that in this setting, as in the additive case, either item pricing or bundle pricing gives a constant-factor approximation to the optimal revenue. ${ }^{3}$ There are many similarities between the two lines of work on unit-demand buyers and additive buyers, and Rubinstein and Weinberg's result can be seen as a unification of the two approaches, albeit with a worse approximation factor.

Multi-parameter settings with multiple buyers are less well understood. For settings with many unit-demand buyers, Chawla et al. [2010a,b] developed a generic approach for approximation via sequential posted-price mechanisms (SPMs). SPMs approach buyers in some predetermined order and offer items for sale to each buyer at predetermined prices while supplies last. For settings with many additive-value buyers, Yao [2015] showed that either running a second-price auction for each item separately or optimally selling to bidder $i$ the set of items for which he is the highest bidder ${ }^{4}$ achieves a constant-factor approximation. Cai et al. [2016] presented a new uniform framework that can be used to rederive both Yao and Chawla et al.'s results, with a tighter analysis for the former. However, prior to our work, no approximations were known for other constrained additive settings or for settings with heterogeneous buyers. Consider, for example, a setting with some unit-demand and some additive buyers. In this case, neither of the results mentioned above provide an approximation. Chawla et al.'s analysis relies on a reduction from multi-dimensional incentive constraints to singledimensional ones that applies only to the unit-demand setting, and, in particular, cannot account for revenue from bundling, which is crucial in non-unit-demand settings. Yao's approach on the other hand relies on allocating each item to the highest value agent, and cannot provide a constant-factor approximation for subadditive agents. ${ }^{5}$

A different approach to optimal mechanism design due to Cai et al. [2012a,b, $2013 \mathrm{a}, \mathrm{b}]$ uses linear programming formulations for settings with small support type distributions, and shows that optimal mechanisms are virtual welfare maximizers. This approach is unsuitable for our setting which, even upon discretization of values, involves distributions over exponential size supports. Moreover, mechanisms generated by this approach tend to lack the nice structure and simplicity of pricingbased mechanisms.

Finally, a new approach to mechanism design has emerged in recent years that uses duality theory to design as well as analyze optimal or approximately optimal mechanisms [see, e.g., Cai et al. 2016; Daskalakis et al. 2015; Giannakopoulos and Koutsoupias 2014, 2015; Haghpanah and Hartline 2015]. Designing good dual solutions in this context, however, involves more art than science, and for the most part, positive results are restricted to very special classes of value functions and value distributions.

\subsection{Our Techniques and Contributions}

Ex Ante Relaxation. Our work follows a generic approach developed by Alaei [2011] for transforming multi-agent mechanism design problems into their single-agent counterparts via the so-called ex ante relaxation. In a multi-agent setting, agents impose

\footnotetext{
${ }^{3}$ Rubinstein and Weinberg [2015]'s result holds for a much broader setting with a single subadditive value agent, but their factor of approximation is rather large - about 340 .

${ }^{4}$ In the latter case, Yao approximates the optimal revenue via two-part tariffs.

${ }^{5}$ To see why Yao's approach cannot work for unit-demand agents, observe that if a single unit-demand agent has the highest value for each item, the seller must try to sell all but one item to non-highest-value buyers in order to obtain good revenue.
} 
externalities upon each other through the seller's supply constraint: each item must be sold to at most one buyer ex post. Alaei proposes relaxing the problem by enforcing the supply constraint ex ante rather than ex post: the probabilities with which an item is sold to the different buyers should sum up to no more than one. In other words, in expectation the item is sold at most once. Applying the ex ante relaxation to a mechanism design problem with multiple buyers involves three steps:

(1) Decompose into single-agent problems: determine the ex ante probabilities with which each item can be sold to each buyer; for each item these probabilities should sum up to no more than 1 ;

(2) Solve single-agent problems: for each agent, find an approximately optimal mechanism satisfying the ex ante supply constraint determined in the first step;

(3) Stitch single-agent mechanisms: combine the single-agent mechanisms developed in the second step into a single mechanism that satisfies the supply constraints ex post.

The first step is conceptually simple and applies in any setting where buyers have independent values. We reproduce this argument in Section 4 for completeness.

Alaei described how to implement the second and third steps for problems involving unit-demand agents. ${ }^{6}$ For the third "stitching" step, he suggested composing the singleagent mechanisms sequentially (similar to the approach of Chawla et al. [2010a]). However, this does not work for arbitrary single-agent mechanisms. Once the composite mechanism has sold off a few items, fewer bundles are available to subsequent buyers, and the mechanism may obtain far less revenue than its single-agent counterparts. We show that two-part tariffs compose well without much loss in revenue when each buyer's value function is additive up to a matroid feasibility constraint (and, more generally, when the value functions satisfy the gross substitutes condition).

Core-Tail Decomposition. In order to bound the single-agent revenue as required in step two of the ex ante approach, we use the core-tail decomposition of $\mathrm{Li}$ and Yao [2013], and its extensions due to Babaioff et al. [2014] and Rubinstein and Weinberg [2015]. Roughly speaking, in the absence of ex ante supply constraints, for any vector of item values, we can partition items into those with small value and those with large value. This partitioning is done in such a manner that the set of large-value items (a.k.a. the tail) contains only a few items in expectation; the revenue generated by these items behaves essentially like unit-demand revenue, and can be recovered by selling the items separately via an argument of Chawla et al. [2010b]. The set of small-value items (a.k.a. the core), on the other hand, displays concentration of value and the revenue generated by these items can be recovered via bundling [Rubinstein and Weinberg 2015].

Under an ex ante supply constraint the revenue generated by the tail can still be recovered via item pricing as before. Bounding the revenue from the core is trickier, however, because different items may face very different ex ante constraints, and their total values may not concentrate well. Furthermore, selling the grand bundle allocates all items with the same probability to the buyer and consequently may not respect the given ex ante constraint. We make a careful choice of thresholds for partitioning values into the core and the tail in such a manner that we can recover the value of the core in two parts: (1) when the ex ante constraint is strong (i.e. the allocation probabilities are mostly small), selling separately recovers most of the core revenue; (2) when the ex ante constraint is weak (i.e. the allocation probabilities are mostly large), bundling

\footnotetext{
${ }^{6}$ Alaei also presented solutions for certain additive-value settings under the assumption that the agents' type spaces are small and given explicitly.
} 
as part of a two-part tariff recovers most of the core revenue while continuing to respect the ex ante constraint.

Prophet inequalities. Observe that the ex ante approach described above relaxes the seller's supply constraint, but continues to enforce the buyer's demand constraint ${ }^{7}$ ex post. It is unclear how a relaxation of the buyer's demand constraint would capture revenue due to bundling, and whether such a relaxation is useful for mechanism design. Nevertheless, our analysis gives rise to a term which corresponds to item-pricing revenue from a common relaxation of the seller's and buyer's constraints. Roughly speaking, this term captures the total revenue that the seller can obtain from the buyer by selling each item separately subject to a bound on the probability of sale, under the condition that these bounds respect both the seller's and the buyer's feasibility constraints in an ex ante sense. For example, for a unit-demand buyer, the probabilities of sale over the items must sum up to no more than 1 . We then employ a prophet inequality to relate this term to the optimal item-pricing revenue for that buyer. A prophet inequality in this context specifies an item pricing that, regardless of which maximal feasible set of items the buyer purchases, obtains in expectation a constant fraction of the ex ante optimal revenue. Prophet inequalities of the above form are known to hold for several classes of feasibility constraints, such as uniform matroids, partition matroids, and their intersections (see, e.g., Chawla et al. 2010a). For general matroid constraints, it is not known whether a prophet inequality with static item prices as described above can obtain a constant approximation factor. ${ }^{8}$ However, Feldman et al. [2016] give a prophet inequality that obtains a constant approximation by restricting the buyer's demand - in other words, by forbidding the buyer to purchase certain feasible sets. We discuss and use these results in Section 5.

The final mechanism. As mentioned earlier, our final mechanism is a sequential twopart tariff mechanism. We remark that buyers in our mechanism are required to pay the entry fee before finding out whether their favorite items will be available when it is their turn to buy; therefore, our mechanism is only Bayesian incentive compatible (BIC), and not necessarily dominant strategy incentive compatible (DSIC). We leave open the question of whether it is possible to approximate the optimal revenue within a constant factor via a DSIC mechanism. In some settings, our mechanism restricts the subsets of items that a buyer is allowed to buy; we call such a mechanism a demand-limiting sequential two-part tariff. This is seen, for instance, in market-style CSA programs in which members can buy only certain quantities and combinations of produce.

Other contributions. As special cases of our general result, we also obtain improvements to the results of Rubinstein and Weinberg [2015]. Recall that Rubinstein and Weinberg show that for a single buyer with subadditive values, either item pricing or bundle pricing obtains a constant-factor approximation. We improve this result in two ways. First, for constrained additive values, we improve the approximation factor from about 340 to 31.1 (Corollary 3.6) ${ }^{9}$ Second, we show that the result holds also under an ex ante constraint for a suitable definition of item pricings and bundle pricings that respect the same ex ante constraint (see Corollary 3.7). Finally, for revenue maximization with multiple additive buyers, we adapt arguments from [Babaioff et al. 2014] to

\footnotetext{
${ }^{7}$ The buyer's demand constraint refers to, e.g., whether the buyer desires one item as in the unit-demand case, or all items as in the additive case.

${ }^{8}$ Kleinberg and Weinberg [2012] present a prophet inequality with adaptive prices, but this is unsuitable for our setting.

${ }^{9}$ It is possible to use Rubinstein and Weinberg's techniques to obtain a better approximation for the special case of constrained additive values, however, the resulting bound is still much weaker than ours.
} 
obtain an approximation factor of 28 (see the full version of this paper [Chawla and Miller 2016]); this is an improvement over Yao [2015]'s approximation factor of 69 for the same setting, but is worse than Cai et al. [2016]'s improvement of Yao's analysis to an 8-approximation. Arguably, our analysis for this setting is conceptually simpler than both of those works.

Symmetric settings. In an interesting special case of our setting, the buyers are a priori symmetric (but items are heterogeneous). That is, each buyer has a value vector drawn from identical independent distributions, and also desires the same bundles of items. In this setting, our mechanism sets the same entry fee as well as item prices for all buyers. Furthermore, these fees and prices can be computed efficiently [Chawla and Miller 2016].

Further directions. For settings with asymmetric buyers, we leave open the question of efficiently solving the ex ante relaxation. Our main result requires buyers' demand constraint to be matroids for two reasons: this allows us to use a prophet inequality for a single agent, and it also enables us to combine single-agent mechanisms sequentially without much loss in revenue. It is an interesting challenge to apply the ex ante approach for demand constraints beyond matroids, or for more general classes of subadditive values.

\section{PRELIMINARIES}

We consider a setting with a single seller and $n$ buyers. The seller has $m$ heterogeneous items to sell. Each buyer $i \in[n]$ has a type composed of a public downward-closed demand constraint $\mathcal{F}_{i} \subseteq 2^{[m]}$ and a private value vector $\mathbf{v}_{i}=\left(v_{i 1}, \cdots, v_{i m}\right)$ that maps items to non-negative values. Roughly speaking, the demand constraint $\mathcal{F}_{i}$ describes the maximal sets of items from which the buyer derives value. Formally, the buyer's value for a set of items is described by a constrained additive function: for $S \subseteq 2^{[m]}$,

$$
\mathbf{v}_{i}(S)=\max _{S^{\prime} \in \mathcal{F}_{i} ; S^{\prime} \subseteq S} \sum_{j \in S^{\prime}} v_{i j}
$$

It will sometimes be necessary to consider feasibility restricted to subsets of the available items. For $M^{\prime} \subseteq[m]$, the restriction of $\mathcal{F}_{i}$ to $M^{\prime}$, denoted $\left.\mathcal{F}_{i}\right|_{M^{\prime}}$, is formed by dropping items not in $M^{\prime}$. Formally, $\left.\mathcal{F}_{i}\right|_{M^{\prime}}=\mathcal{F}_{i} \cap 2^{M^{\prime}}$. We will typically assume that for all $i, \mathcal{F}_{i}$ is a matroid; see Appendix A for a review of matroid concepts.

We assume that the values $v_{i j}$ are drawn from distribution $\mathcal{D}_{i j}$ independently of all other values; we use $\mathcal{D}_{i}=\prod_{j} \mathcal{D}_{i j}$ to denote the joint distribution of buyer $i$ 's value vector and $\mathcal{D}=\prod_{i} \mathcal{D}_{i}$ to denote the joint distribution over all value vectors. The demand constraints $\mathcal{F}_{i}$ may be different for different buyers. Let $\mathcal{F}=\left\{\mathcal{F}_{i}\right\}_{i \in[n]}$ denote the tuple of feasibility constraints, one for each buyer.

\subsection{Incentive Compatible Mechanisms and Revenue Maximization}

A mechanism $\mathcal{M}$ takes as input the value vectors $\mathbf{v}=\left(\mathbf{v}_{1}, \cdots, \mathbf{v}_{n}\right)$ and returns an allocation $\mathbf{x}(\mathbf{v})$ and payment vector $\mathbf{p}(\mathbf{v})$. Here $\mathbf{x}_{i}(\mathbf{v})$ denotes the (potentially random) set of items that is allocated to buyer $i$. A mechanism $\mathcal{M}$ is supply-feasible if every item is allocated to at most one buyer; in other words, for all $\mathbf{v}$, and $i_{1} \neq i_{2}, \mathbf{x}_{i_{1}}(\mathbf{v}) \cap \mathbf{x}_{i_{2}}(\mathbf{v})=\emptyset$ with probability 1 .

We use $x_{i j}(\mathbf{v})$ to denote the probability with which buyer $i$ receives item $j$. Without loss of generality, we focus on mechanisms that for every value vector $\mathbf{v}$ and every buyer $i$ satisfy $\mathbf{x}_{i}(\mathbf{v}) \in \mathcal{F}_{i}$ with probability 1 ; we call such mechanisms demand-feasible. Consequently, we note that the vector $\left(x_{i 1}(\mathbf{v}), \cdots, x_{i m}(\mathbf{v})\right)$ lies in the polytope enclosing 
$\mathcal{F}_{i}$, which we denote ${ }^{10} \mathcal{P}_{\mathcal{F}_{i}}$. In the rest of the paper we will overload notation and use $\mathbf{x}_{i}(\mathbf{v})$ to denote the vector $\left(x_{i 1}(\mathbf{v}), \cdots, x_{i m}(\mathbf{v})\right)$.

We assume that buyers are risk neutral and have quasi-linear utilities. In other words, the utility that a buyer derives from allocation $\mathbf{x}_{i}$ and payment $\mathbf{p}_{i}$ is given by $\mathbf{x}_{i} \cdot \mathbf{v}_{i}-\mathbf{p}_{i}$. We consider mechanisms which are Bayesian incentive compatible (BIC). A mechanism is BIC if truthtelling is a Bayes-Nash equilibrium; that is, if a buyer maximizes his own utility -in expectation over other buyers' values, assuming they report truthfully, as well as randomness inherent in the mechanism-by reporting truthfully. In contrast, a mechanism is dominant-strategy incentive compatible (DSIC) if truthtelling is a dominant strategy; that is, if a buyer maximizes his own utility by reporting truthfully, regardless of what other buyers report.

We are interested in revenue maximization for the seller. The seller's revenue from a BIC mechanism $\mathcal{M}=(\mathbf{x}, \mathbf{p})$ at value vectors $\mathbf{v}$ is $\sum_{i} \mathbf{p}_{i}(\mathbf{v})$, and the expected revenue is $\operatorname{REV}^{\mathcal{M}}(\mathcal{D})=\mathbf{E}_{\mathbf{v} \sim \mathcal{D}}\left[\sum_{i} \mathbf{p}_{i}(\mathbf{v})\right]$. The revenue maximization problem seeks to maximize $\operatorname{REV}^{\mathcal{M}}(\mathcal{D})$ over all BIC mechanisms that are demand- and supply-feasible; we use $\operatorname{REV}(\mathcal{D}, \mathcal{F})$ to denote this maximum revenue.

\subsection{Ex Ante Constrained Revenue Maximization}

We will reduce the multiple buyer revenue maximization problem described above to single-buyer problems with ex ante supply constraints. The following definitions are for a single agent $i$; we omit the subscript $i$ for clarity. Let $\mathbf{q}=\left(q_{1}, \cdots, q_{n}\right)$ be a vector of probabilities with $q_{j} \in[0,1]$ for all $j \in[m]$. A mechanism $\mathcal{M}=(\mathbf{x}, \mathbf{p})$ is q-constrained under $\mathcal{D}$ if for all items $j \in[m]$, its ex ante probability for selling item $j$ when values are drawn from $\mathcal{D}, \mathbf{E}_{\mathbf{v} \sim \mathcal{D}}\left[x_{j}(\mathbf{v})\right]$, is at most $q_{j}$. We will consider both revenue and welfare maximization problems over q-constrained mechanisms. Formally, we define

$$
\operatorname{REV}_{\mathbf{q}}(\mathcal{D}, \mathcal{F})=\max _{\mathcal{M}=(\mathbf{x}, \mathbf{p}): \mathbf{E}_{\mathbf{v} \sim \mathcal{D}}\left[x_{j}(\mathbf{v})\right] \leq q_{j} \forall j \in[m]} \operatorname{REV}^{\mathcal{M}}(\mathcal{D})
$$

and

$$
\operatorname{VAL}_{\mathbf{q}}(\mathcal{D}, \mathcal{F})=\max _{\mathcal{M}=(\mathbf{x}, \mathbf{p}): \mathbf{E}_{\mathbf{v} \sim \mathcal{D}}\left[x_{j}(\mathbf{v})\right] \leq q_{j} \forall j \in[m]} \operatorname{VAL}^{\mathcal{M}}(\mathcal{D}),
$$

where the maximum is taken over all BIC demand-feasible mechanisms ${ }^{11}$ and $\operatorname{VAL}^{\mathcal{M}}(\mathcal{D})=\mathbf{E}_{\mathbf{v} \sim \mathcal{D}}[\mathbf{x}(\mathbf{v}) \cdot \mathbf{v}]$.

It will sometimes be convenient to express the ex ante constraint in the form of ex ante prices defined as: $\beta_{j}=\mathcal{D}_{j}{ }^{-1}\left(1-q_{j}\right)$. In other words, for every $j \in[m], \beta_{j}$ is defined such that the probability that $v_{j}$ exceeds this price is precisely $q_{j}$. Note that there is a one-one correspondence between ex ante probabilities and ex ante prices.

\subsection{Special Single-Agent Mechanisms}

Item pricing. An item pricing is defined by a set of prices $p_{j}$, one for each item $j$. A buyer is allowed to select as many items as he pleases, up to some downward-closed constraint $\mathcal{F}$, and he pays the sum of the associated prices. That is, if the buyer selects the set $S \subseteq[m]$, he pays $\sum_{j \in S} p_{j}$. The buyer then selects the set $S \in \mathcal{F}$ which maximizes $\sum_{j \in S}\left(v_{j}-p_{j}\right)$. We use $\operatorname{SREv}(\mathcal{D}, \mathcal{F})$ to denote the optimal revenue obtainable by any item pricing from a buyer with value distribution $\mathcal{D}$ and demand constraint $\mathcal{F}$.

\footnotetext{
${ }^{10}$ Formally, $\mathcal{P}_{\mathcal{F}_{i}}$ is the convex hull of the incidence vectors of all sets in $\mathcal{F}_{i}$ in $\Re^{m}$.

${ }^{11}$ We don't need to impose the supply-feasibility constraint explicitly — this is already implicit in the ex ante probability constraint.
} 
Bundle pricing. A bundle pricing is defined by a single price (a.k.a. entry fee) $\pi$. A buyer can buy any subset of items satisfying the demand constraint $\mathcal{F}$ at price $\pi$. A rational buyer chooses to participate (i.e. pay the fee) if $v([m])=\max _{S \in \mathcal{F}} v(S) \geq \pi$ and then selects a corresponding maximal set $S$. We use $\operatorname{BREV}(\mathcal{D}, \mathcal{F})$ to represent the optimal revenue obtainable by any bundle pricing from a buyer with value distribution $\mathcal{D}$ and demand constraint $\mathcal{F}$.

Two-part tariffs. A two-part tariff is a common generalization of both item pricings and bundle pricings. It is described by an $m+1$ dimensional vector of prices: $\left(\pi, p_{1}, \cdots, p_{m}\right)$. The mechanism offers each set $S \subseteq[m]$ of items to the buyer at a price of $\pi+\sum_{j \in S} p_{j}$; the buyer can then choose to buy his favorite set at these offered prices. Informally speaking, the mechanism charges the buyer an entry fee of $\pi$ for the right to buy any set of items, with item $j$ offered at a fixed price of $p_{j}$. Like other pricing-based mechanisms, two-part tariffs are deterministic, dominant strategy incentive compatible mechanisms.

A utility-maximizing buyer with values $\mathbf{v}$ and feasibility constraint $\mathcal{F}$ when offered a two-part tariff $(\pi, \mathbf{p})$ buys the set $S \in \mathcal{F}$ of items that maximizes $\mathbf{v}(S)-\pi-\sum_{j \in S} p_{j}$, if that quantity is non-negative ${ }^{12}$; in that case, we say that the buyer participates in the mechanism. We denote the revenue of a two-part tariff $(\pi, \mathbf{p})$ offered to a buyer with feasibility constraint $\mathcal{F}$ and value distribution $\mathcal{D}$ by $\operatorname{REV}^{(\pi, \mathbf{p})}(\mathcal{D}, \mathcal{F})$. We use $\operatorname{TREv}(\mathcal{D}, \mathcal{F})$ to denote the optimal revenue that a two-part tariff can obtain from a buyer with value distribution $\mathcal{D}$ and demand constraint $\mathcal{F}$.

Two-part tariffs are known to be approximately optimal in certain single-agent settings. The following results ${ }^{13}$ are due to Chawla et al. [2010b] and Babaioff et al. [2014] respectively. Rubinstein and Weinberg [2015] proved a similar result for constrained additive values, but with a very large approximation factor (about 340 ).

$$
\begin{gathered}
\operatorname{ReV}\left(\mathcal{D}, \mathcal{F}_{\text {UnitDemand }}\right) \leq 4 \operatorname{TREV}\left(\mathcal{D}, \mathcal{F}_{\text {Unitdemand }}\right) \\
\operatorname{REV}\left(\mathcal{D}, \mathcal{F}_{\text {Additive }}\right) \leq 6 \operatorname{TREV}\left(\mathcal{D}, \mathcal{F}_{\text {Additive }}\right)
\end{gathered}
$$

Pricings with an ex ante constraint. Next we extend the above definitions to respect ex ante supply constraints. We say that a two-part tariff $(\pi, \mathbf{p})$ satisfies ex ante constraint $\mathbf{q}$ if for all $j, p_{j} \geq \beta_{j}=\mathcal{D}_{j}{ }^{-1}\left(1-q_{j}\right)$. Note that this is a stronger condition than merely requiring that the mechanism allocates item $j$ with ex ante probability at most $q_{j}$. We use $\operatorname{TREV}_{\mathbf{q}}(\mathcal{D}, \mathcal{F})$ to denote the optimal revenue achieved by a demand-feasible two-part tariff that satisfies ex ante constraint q. Likewise, we use $\operatorname{SREV}_{\mathbf{q}}(\mathcal{D}, \mathcal{F})$ to denote the optimal revenue achievable by an item pricing $\mathbf{p}$ with $p_{j} \geq \beta_{j}$ for all $j$.

\subsection{Multi-Agent (Sequential) Two-Part Tariff Mechanisms}

We now extend the definition of two-part tariffs to multi-agent settings. Consider a setting with $n$ agents and demand constraints $\mathcal{F}=\left\{\mathcal{F}_{i}\right\}_{i \in[n]}$. A sequential two-part tariff for this setting is parameterized by an ordering $\sigma$ over the agents, a set of entry fees $\pi=\left(\pi_{1}, \cdots, \pi_{n}\right)$, and a set of prices $\mathbf{p}=\left\{p_{i j}\right\}$. The mechanism proceeds as follows.

(1) The ordering $\sigma$ and prices $\pi ; \mathbf{p}$ are announced.

(2) Each agent $i$ independently decides whether or not to participate in the mechanism. If the agent decides to participate, then he pays his corresponding entry fee $\pi_{i}$.

\footnotetext{
${ }^{12}$ This is essentially an ex-post IR condition.

${ }^{13}$ Here $\mathcal{F}_{\text {UnitDemand }}=\{S \subset[m]|| S \mid=1\}$ represents a unit-demand buyer, and $\mathcal{F}_{\text {Additive }}=2^{[m]}$ represents a buyer with fully additive values.
} 
(3) The mechanism considers agents in the order given by $\sigma$. When an agent $i$ is considered, if the agent previously declined to participate, no items are allocated and no payment is charged. Otherwise, of the items unallocated so far, the agent is allowed to purchase his favorite feasible set of items at the prices $p_{i j}$.

Observe that agents choose whether or not to participate in the mechanism before knowing which items will be available when it is their turn to purchase. Accordingly, a sequential two-part tariff is BIC but not necessarily DSIC.

The sequential two-part tariff mechanisms that we develop in this paper are order oblivious in the sense that their revenue guarantees hold regardless of the ordering $\sigma$ chosen over the agents. Accordingly, in describing these mechanisms, we need only specify the prices $\pi ; \mathbf{p}$.

In some cases, our two-part tariff mechanisms disallow agents from buying certain sets of items. Specifically, a demand-limiting sequential two-part tariff is parameterized by an ordering $\sigma$, prices $\pi$; $\mathbf{p}$, as well as feasibility constraints $\mathcal{F}^{\prime}=\left\{\mathcal{F}_{i}^{\prime}\right\}_{i \in[n]}$ where, for every agent $i, \mathcal{F}_{i}^{\prime} \subseteq \mathcal{F}$ is a matroid constraint stronger than the agent's original demand constraint. When it is agent $i$ 's turn to buy items, the agent is allowed to buy any subset of items in $\mathcal{F}_{i}^{\prime}$. In particular, the agent is not allowed to buy sets of items in $\mathcal{F}_{i} \backslash \mathcal{F}_{i}^{\prime}$.

\section{MAIN RESULTS}

We now state our three main results corresponding to the three parts of the ex ante approach for approximating $\operatorname{REV}(\mathcal{D}, \mathcal{F})$. Lemma 3.1 corresponds to the first relaxation step, and states that the revenue $\operatorname{REV}(\mathcal{D}, \mathcal{F})$ can be bounded by the sum of single-agent revenues with appropriate ex ante constraints. While the lemma is stated here for buyers with constrained additive values, it holds for arbitrary value functions as long as values are independent across buyers. See Chawla and Miller [2016] for a proof.

Lemma 3.1 (Relaxation). For any feasibility constraints $\mathcal{F}=\left\{\mathcal{F}_{i}\right\}_{i \in[n]}$ and value distributions $\mathcal{D}=\prod_{i} \mathcal{D}_{i}$, there exist ex ante probability vectors $\mathbf{q}_{1}, \cdots, \mathbf{q}_{n}$, satisfying: (1) $\mathbf{q}_{i} \in \mathcal{P}_{\mathcal{F}_{i}}$ for all $i$, and, (2) $\sum_{i} q_{i j} \leq 1$ for all $j$, such that

$$
\operatorname{REV}(\mathcal{D}, \mathcal{F}) \leq \sum_{i} \operatorname{REv}_{\mathbf{q}_{i}}\left(\mathcal{D}_{i}, \mathcal{F}_{i}\right)
$$

Lemma 3.2 corresponds to the last stitching step, and shows that any single-agent two-part tariff mechanisms that collectively satisfy an ex ante constraint on every item can be stitched together into a multi-agent sequential two-part tariff mechanism without losing much revenue.

LEMMA 3.2. For every agent $i$, let $\mathcal{M}_{i}=\left(\pi_{i}, \mathbf{p}_{i}\right)$ be any two-part tariff that is demand-feasible with respect to a matroid feasibility constraint $\mathcal{F}_{i}$ and that satisfies ex ante supply constraints $\mathbf{q}_{i}$ under value distribution $\mathcal{D}_{i}$. Let $\mathcal{F}=\left\{\mathcal{F}_{i}\right\}_{i \in[n]}$ and $\mathcal{D}=\prod_{i} \mathcal{D}_{i}$. Then, if $\sum_{i} q_{i j} \leq 1 / 2$ for all $j$, there exists a sequential two-part tariff mechanism $\mathcal{M}$ that is supply-feasible and demand-feasible with respect to $\mathcal{F}$ such that

$$
\operatorname{REV}^{\mathcal{M}}(\mathcal{D}) \geq \frac{1}{2} \sum_{i} \operatorname{REV}^{\mathcal{M}_{i}}\left(\mathcal{D}_{i}\right)
$$

We therefore obtain the following corollary.

Corollary 3.3 (Stitching). For any value distributions $\mathcal{D}=\prod_{i} \mathcal{D}_{i}$ and feasibility constraints $\mathcal{F}=\left\{\mathcal{F}_{i}\right\}_{i \in[n]}$, where each $\mathcal{F}_{i}$ is a matroid, let $\mathbf{q}_{1}, \cdots, \mathbf{q}_{n}$ be any ex ante probability vectors satisfying $\sum_{i} q_{i j} \leq 1 / 2$ for all $j$. Then, there exists a demand-and 
supply-feasible sequential two-part tariff mechanism $\mathcal{M}$ such that

$$
\operatorname{REV}^{\mathcal{M}}(\mathcal{D}) \geq \frac{1}{2} \sum_{i} \operatorname{TREV}_{\mathbf{q}_{i}}\left(\mathcal{D}_{i}, \mathcal{F}_{i}\right)
$$

In order to put together the Relaxation Lemma and the Stitching Corollary, it remains to relate $\operatorname{REV}_{\mathbf{q}}$ for a single agent to $\mathrm{TREV}_{\mathbf{q}}$ for the same agent. The following lemma presents such a relationship when the buyer's demand constraint is a matroid.

LEMMA 3.4 (Single-agent approximation). Let $\mathcal{D}$ be any product value distribution and $\mathcal{F}$ be a matroid with feasible polytope $\mathcal{P}_{\mathcal{F}}$. Then, for any $q \in \frac{1}{2} \mathcal{P}_{\mathcal{F}}$, there exists a submatroid $\mathcal{F}^{\prime} \subseteq \mathcal{F}$ such that

$$
\operatorname{REV}_{\mathbf{q}}(\mathcal{D}, \mathcal{F}) \leq 33.1 \operatorname{TREV}_{\mathbf{q}}\left(\mathcal{D}, \mathcal{F}^{\prime}\right)
$$

If $\mathcal{F}$ is a partition matroid, then $\mathcal{F}^{\prime}=\mathcal{F}$.

Putting Lemmas 3.1 and 3.4, and Corollary 3.3 together, and observing that by the concavity of the revenue objective, $\operatorname{REV}_{\frac{1}{2} \mathbf{q}_{i}}\left(\mathcal{D}_{i}, \mathcal{F}_{i}\right) \geq \frac{1}{2} \operatorname{REV}_{\mathbf{q}_{i}}\left(\mathcal{D}_{i}, \mathcal{F}_{i}\right)$ for all $i$, we get our main result.

THEOREM 3.5. For any product value distribution $\mathcal{D}$ and feasibility constraints $\mathcal{F}=\left\{\mathcal{F}_{i}\right\}_{i \in[n]}$, where each $\mathcal{F}_{i}$ is a matroid, there exist submatroids $\mathcal{F}_{i}^{\prime} \subseteq \mathcal{F}_{i}$ and a supply-feasible $\left\{\mathcal{F}_{i}^{\prime}\right\}$-limited sequential two-part tariff mechanism $\mathcal{M}$ such that

$$
\operatorname{REV}(\mathcal{D}, \mathcal{F}) \leq 133 \operatorname{REV}^{\mathcal{M}}(\mathcal{D})
$$

If $\mathcal{F}_{i}$ is a partition matroid, then $\mathcal{F}_{i}^{\prime}=\mathcal{F}_{i}$.

Further results. As a consequence of our single-agent approximation (Lemma 5.6 in Section 5), we also obtain an improved approximation for the single-agent revenue maximization problem with constrained additive values. Specifically, taking $q=\overrightarrow{\mathbf{1}}$ and noting $\beta=\overrightarrow{0}$, Lemma 5.6 gives the following bound on the optimal revenue for the single-agent setting.

COROLlARY 3.6. For any downward closed feasibility constraint $\mathcal{F}$ and any value distribution $\mathcal{D}$,

$$
\operatorname{Rev}(\mathcal{D}, \mathcal{F}) \leq 31.1 \max \{\operatorname{SREv}(\mathcal{D}, \mathcal{F}), \operatorname{BREV}(\mathcal{D}, \mathcal{F})\} .
$$

Also as a consequence of Lemma 5.6, we show the following bound for revenue maximization under an arbitrary ex ante constraint.

COROLlaRY 3.7. Let $\mathcal{D}$ be any product value distribution and $\mathcal{F}$ be a matroid. Then for any $q \in[0,1]^{m}$, there exists a submatroid $\mathcal{F}^{\prime} \subseteq \mathcal{F}$ such that

$$
\operatorname{REV}_{\mathbf{q}}(\mathcal{D}, \mathcal{F}) \leq 35.1 \operatorname{TREV}_{\mathbf{q}}\left(\mathcal{D}, \mathcal{F}^{\prime}\right)
$$

If $\mathcal{F}$ is a partition matroid, then $\mathcal{F}^{\prime}=\mathcal{F}$.

\section{THE EX ANTE RELAXATION AND STITCHING}

In this section we prove Lemma 3.2.

Proof of Lemma 3.2. For every buyer $i$, let $\pi_{i}$ and $\left(p_{i 1}, \cdots, p_{i m}\right)$ denote the entry fee and item prices respectively in the mechanism $\mathcal{M}_{i}$. We will compose the mechanisms $\mathcal{M}_{i}$ to obtain a single mechanism $\mathcal{M}$ as follows.

The mechanism $\mathcal{M}$ considers buyers in an arbitrary order and offers items for sale sequentially to the buyers in that order. When it is buyer $i$ 's turn, some (random set of) items have already been sold to other buyers. The mechanism offers the remaining 
items to buyer $i$ via a two-part tariff: it charges the buyer an entry fee of $\frac{1}{2} \pi_{i}$ for the right to buy any subset of the remaining items, with item $j$ priced at $p_{i j}$. Importantly, buyers must make the decision of whether or not to participate (that is, whether or not to pay the entry fee) before knowing which items are left unsold.

By definition, the mechanism is BIC: buyers may choose whether or not to participate and which subset of items to purchase.

Let us now consider a single buyer $i$. We first claim that when the mechanism $\mathcal{M}$ considers buyer $i$, for every item $j$, the probability (taken over value vectors of other agents) that item $j$ is available to be bought by $i$ is at least $1 / 2$. Recall that for every pair $i, j, \mathbf{P r}_{v_{i j} \sim \mathcal{D}_{i j}}\left[v_{i j}>p_{i j}\right]=1-\mathcal{D}_{i j}\left(p_{i j}\right) \leq 1-\mathcal{D}_{i j}\left(\beta_{i j}\right)=q_{i j}$. So the probability that some agent $i^{\prime}$ buys item $j$ is at most $q_{i^{\prime} j}$. Therefore, the probability (over values of agents other than $i$ ) that item $j$ is allocated to an agent other than $i$ is at most $\sum_{i^{\prime} \neq i} q_{i^{\prime} j} \leq 1 / 2$, and this proves the claim.

We will now use the above claim to argue that if after drawing his value vector the buyer chooses to participate (i.e. pay the entry fee) in mechanism $\mathcal{M}_{i}$, then he chooses to participate in $\mathcal{M}$. If agent $i$ participates in mechanism $\mathcal{M}_{i}$, then for some set $S \in \mathcal{F}_{i}$ his value vector satisfies $\sum_{j \in S}\left(v_{i j}-p_{i j}\right)-\pi_{i}>0$. In the mechanism $\mathcal{M}$, the agent derives from the same set $S$ an expected utility of

$$
\left(\sum_{j \in S} \mathbf{P r}[j \text { is available for } i]\left(v_{i j}-p_{i j}\right)\right)-\frac{1}{2} \pi_{i},
$$

which by the above claim is at least $1 / 2\left(\sum_{j \in S}\left(v_{i j}-p_{i j}\right)-\pi_{i}\right)>0$. Consequently, if $\mathcal{M}_{i}$ obtains the entry fee $\pi_{i}$ from agent $i$, then $\mathcal{M}$ obtains the entry fee $\pi_{i} / 2$.

Next we claim that if agent $i$ buys item $j$ in mechanism $\mathcal{M}_{i}$ and item $j$ is available for him in mechanism $\mathcal{M}$, then the agent buys item $j$ in $\mathcal{M}$. This follows directly from Lemma A.1 (Appendix A) by noting that $\mathcal{M}_{i}$ and $\mathcal{M}$ offer the same item prices to the agent and that the agent is a utility maximizer. As argued previously, item $j$ is available with probability at least $1 / 2$, therefore, this claim implies that if $\mathcal{M}_{i}$ obtains the price $p_{i j}$ from agent $i$, then $\mathcal{M}$ obtains the same price $p_{i j}$ with probability $1 / 2$. Putting this together with the above observation about entry fee, we get that $\mathcal{M}$ obtains in expectation at least half of the total revenue obtained by the mechanisms $\mathcal{M}_{i}$.

The proof of the lemma relies upon three facts: (1) mechanism $\mathcal{M}$ offers each item with probability at least half to each buyer, (2) under these probabilities, the buyer's expected utility from a set $S$ is at least half his utility from obtaining $S$ with certainty, and, (3) in the composed mechanism, the buyer selects those items in $S$ that are still available. Fact (2) holds more generally for a buyer with any monotone submodular value function [Feige et al. 2011]. Fact (3) holds for any gross substitutes valuation, a special case of submodular value functions. So Lemma 3.2 holds more generally for buyers with GS valuations.

\section{TWO-PART TARIFFS FOR A SINGLE AGENT}

We now turn to bounding the revenue from a single agent subject to an ex ante constraint. In this section we will prove Lemma 3.4. In the following discussion, we assume that the buyer has a product value distribution $\mathcal{D}=\prod_{j} \mathcal{D}_{j}$, and faces a demand feasibility constraint $\mathcal{F}$, while the mechanism is subject to an ex ante supply constraint q. Recall that we define the ex ante prices $\beta$ as $\beta_{j}=\mathcal{D}_{j}^{-1}\left(1-q_{j}\right)$ for all items $j$.

Core-tail decomposition with ex ante constraints. We begin by defining the notation for the core-tail decomposition (see Table I). Let $\tau \geq 0$ be a constant to be defined later. We use $t_{j}=\beta_{j}+\tau$ to denote the threshold for classifying values into the core or the tail. 
Table I. Notation for Section 5.

\begin{tabular}{rll}
\hline Notation & Definition & Formula \\
\hline $\mathbf{q}$ & Ex ante probabilities & \\
$\beta$ & Ex ante prices & $\beta_{j}=\mathcal{D}_{j}{ }^{-1}\left(1-q_{j}\right) \forall j \in[m]$ \\
$t_{j}$ & Core-tail threshold for item $j$ & $\beta_{j}+\tau$ \\
$\tau$ & Difference between $t_{j}$ and $\beta_{j}$; same for all items & $\min \left\{t \mid \mathbf{P r}_{\mathbf{v} \sim \mathcal{D}}\left[v_{j} \leq t+\beta_{j} \forall j\right] \geq 1 / 2\right\}$ \\
$\mathcal{D}_{j}^{C}$ & Core distribution for item $j$ & $\left.\mathcal{D}_{j}\right|_{v_{j} \leq t_{j}}$ \\
$\mathcal{D}_{j}^{T}$ & Tail distribution for item $j$ & $\left.\mathcal{D}_{j}\right|_{v_{j}>t_{j}}$ \\
$\mathcal{D}_{A}^{C}$ & Core distribution for items not in $A$ & $\prod_{j \notin A} \mathcal{D}_{j}^{C}$ \\
$\mathcal{D}_{A}^{T}$ & Tail distribution for items in $A$ & $\prod_{j \in A} \mathcal{D}_{j}^{T}$ \\
$\xi_{j}$ & Probability item $j$ is in the tail & $\mathbf{P r}_{v_{j} \sim \mathcal{D}_{j}}\left[v_{j}>t_{j}\right]$ \\
$\rho_{A}$ & Probability exactly items in $A$ are in the tail & $\left(\prod_{j \in A} \xi_{j}\right)\left(\prod_{j \notin A}\left(1-\xi_{j}\right)\right)$ \\
$\mathcal{D}-\mathbf{p}$ & Distribution $\mathcal{D}$ shifted to the left by $\mathbf{p}$ & \\
\hline
\end{tabular}

Specifically, for any item $j$, if $v_{j}>t_{j}$, we say item $j$ is in the tail, otherwise it is in the core. Let $\mathcal{D}_{j}^{C}$ (resp., $\mathcal{D}_{j}^{T}$ ) denote the distribution for item $j$ 's value conditioned on the item being in the core (resp., tail).

For a set $A \subseteq[m]$ of items, let $\rho_{A}$ denote the probability that the items in $A$ are in the tail and the remaining items are in the core; that is, $\rho_{A}=$ $\left(\prod_{j \in A} \mathbf{P r}_{v_{j} \sim \mathcal{D}_{j}}\left[v_{j}>t_{j}\right]\right)\left(\prod_{j \notin A} \mathbf{P r}_{v_{j} \sim \mathcal{D}_{j}}\left[v_{j} \leq t_{j}\right]\right)$. Then $\rho_{\emptyset}$ denotes the probability that all items are in the core. Observe that as we increase the constant $\tau$ (thereby increasing the core-tail thresholds uniformly), the probability $\rho_{\emptyset}$ increases. We pick $\tau$ to be the smallest non-negative number such that $\rho_{\emptyset} \geq 1 / 2$. Observe that $\tau>0$ implies $^{14}$ $\rho_{\emptyset}=1 / 2$.

We now state our version of the core-tail decomposition, extended to respect ex ante constraints. We defer the proof to Section 5.4. Note that although the sum over tail revenues does not explicitly enforce the ex ante constraints, the tail distributions are supported only on values above the ex ante prices $\beta$.

Lemma 5.1 (Core Decomposition with Ex Ante Constraints). For any product distribution $\mathcal{D}$, feasibility constraint $\mathcal{F}$, and ex ante constraint $\mathbf{q}$,

$$
\operatorname{REV}_{\mathbf{q}}(\mathcal{D}, \mathcal{F}) \leq \operatorname{VAL}_{\mathbf{q}}\left(\mathcal{D}_{\emptyset}^{C}, \mathcal{F}\right)+\sum_{A \subseteq[m]} \rho_{A} \operatorname{REV}\left(\mathcal{D}_{A}^{T},\left.\mathcal{F}\right|_{A}\right)
$$

\subsection{Bounding the Tail}

We first show that the tail revenue can be bounded by selling items separately under the given ex ante supply constraint $\mathbf{q}$. The main result of this section is as follows.

LEMMA 5.2. For any product distribution $\mathcal{D}$ over $m$ independent items and any $\mathcal{F}$,

$$
\sum_{A \subseteq[m]} \rho_{A} \operatorname{REV}\left(\mathcal{D}_{A}^{T},\left.\mathcal{F}\right|_{A}\right) \leq 8(1+\ln 2) \operatorname{SREV}_{\mathbf{q}}(\mathcal{D}, \mathcal{F})
$$

PRoOF. We make use of the following weak but general relationship between the optimal revenue and the revenue generated by selling separately for a single-agent

\footnotetext{
${ }^{14}$ For simplicity, we are assuming that the value distribution does not contain any point masses; it is easy to modify our argument to work in the absence of this assumption, but we omit the details.
} 
constrained additive value setting; this follows by noting that REV and SREV are within a factor of 4 of each other for unit demand agents (see [Chawla et al. 2010b; Chawla and Miller 2016]).

Claim 1. For any product distribution $\mathcal{D}$ over $m$ items and any $\mathcal{F}$,

$$
\operatorname{REV}(\mathcal{D}, \mathcal{F}) \leq 4 m \operatorname{SREV}\left(\mathcal{D}, \mathcal{F}_{\text {UnitDemand }}\right) \text {. }
$$

Applying this claim to the revenues $\operatorname{REV}\left(\mathcal{D}_{A}^{T},\left.\mathcal{F}\right|_{A}\right)$, we get that $\sum_{A} \rho_{A} \operatorname{REV}\left(\mathcal{D}_{A}^{T},\left.\mathcal{F}\right|_{A}\right) \leq$ $4 \sum_{A} \rho_{A}|A| \operatorname{SREv}\left(\mathcal{D}_{A}^{T}, \mathcal{F}_{\text {UnitDemand }}\right)$. We will now use the fact that the tail contains few items in expectation. Let $\xi_{j}$ denote the probability that item $j$ is in the tail: $\xi_{j}=\mathbf{P r}_{v_{j} \sim \mathcal{D}_{j}}\left[v_{j}>t_{j}\right]$. We can write the following series of inequalities.

$$
\begin{aligned}
\sum_{A} \rho_{A}|A| \operatorname{SREv}\left(\mathcal{D}_{A}^{T}, \mathcal{F}_{\text {Unitdemand }}\right) & \leq \sum_{A} \rho_{A}|A| \sum_{j \in A} \operatorname{ReV}\left(\mathcal{D}_{j}^{T}\right) \\
& =\sum_{j \in[m]} \operatorname{Rev}\left(\mathcal{D}_{j}^{T}\right) \sum_{A \ni j} \rho_{A}|A| \\
& \left.=\sum_{j \in[m]} \xi_{j} \operatorname{REV}\left(\mathcal{D}_{j}^{T}\right) \mathbf{E}|| A|| j \in A\right] \\
& \leq(1+\ln 2) \sum_{j \in[m]} \operatorname{REv}_{\xi_{j}}\left(\mathcal{D}_{j}\right) \\
& \leq \frac{1}{\rho_{\emptyset}}(1+\ln 2) \operatorname{SREv}_{\xi}(\mathcal{D}, \mathcal{F})
\end{aligned}
$$

Here inequality (2) follows by removing the demand constraint $\mathcal{F}_{\text {UnitDemand }}$. Inequality (3) follows from three observations: (1) the tail is non-empty with probability at most $1 / 2$; (2) if $\left\{z_{i}\right\}_{i \in[n]}$ are probabilities satisfying $\prod_{i}\left(1-z_{i}\right) \geq 1 / 2$, then $\sum_{i} z_{i} \leq \ln 2$; (3) a single-agent single-item mechanism for value distribution $\mathcal{D}_{j}^{T}$ that achieves revenue $\operatorname{REV}\left(\mathcal{D}_{j}^{T}\right)$ would achieve $\xi_{j}$ times that revenue on the value distribution $\mathcal{D}_{j}$ while satisfying an ex ante supply constraint of $\xi_{j}$. Inequality (4) follows from the standard argument that the revenue obtained by selling each item individually at prices $t_{j}$ (or higher) is at least $\rho_{\emptyset}$ times the sum of the corresponding per-item revenues. Finally, the result follows by recalling that $\rho_{\emptyset} \geq 1 / 2$ and relaxing the ex ante constraint.

\subsection{Bounding the Core}

Recall that an item $j$ is in the core if its value $v_{j}$ is no more than the threshold $t_{j}=$ $\beta_{j}+\tau$. We will bound the ex ante constrained social welfare of the core, $\operatorname{VAL}_{\mathbf{q}}\left(\mathcal{D}_{\emptyset}^{C}, \mathcal{F}\right)$, in two parts: the welfare obtained from values below $\beta$ via a prophet inequality and the welfare between $\beta$ and $\beta+\tau$ using a concentration bound introduced by Rubinstein and Weinberg [2015].

Recall that $\mathcal{D}_{j}^{C}$ denotes the value distribution for item $j$ conditioned on being in the core. We use $\mathcal{D}_{j}^{C}-\beta_{j}$ to denote the distribution of $v_{j}-\beta$ conditioned on $v_{j}$ being in the core; in other words, $\mathcal{D}_{j}^{C}-\beta_{j}$ is the distribution $\mathcal{D}_{j}^{C}$ shifted to the left by $\beta_{j}$. $\mathcal{D}_{\emptyset}^{C}-\beta$ is defined to be the product of the distributions $\mathcal{D}_{j}^{C}-\beta_{j}$. Observe that value vectors drawn from $\mathcal{D}_{\emptyset}^{C}-\beta$ are bounded by $\tau$ in every coordinate. The following lemma breaks $\operatorname{VAL}_{\mathbf{q}}\left(\mathcal{D}_{\emptyset}^{C}, \mathcal{F}\right)$ up into the two components, each of which can be bounded separately.

LEMMA 5.3. For any product disribution $\mathcal{D}$ and downwards closed feasibility constraint $\mathcal{F}, \operatorname{VAL}_{\mathbf{q}}\left(\mathcal{D}_{\emptyset}^{C}, \mathcal{F}\right) \leq \beta \cdot \mathbf{q}+\operatorname{VAL}\left(\mathcal{D}_{\emptyset}^{C}-\beta, \mathcal{F}\right)$. 
PROOF. Let $x(v)$ be the interim allocation rule of a $q$-constrained BIC mechanism which attains social welfare equal to $\operatorname{VAL}_{\mathbf{q}}\left(\mathcal{D}_{\emptyset}^{C}, \mathcal{F}\right)$. Then

$$
\begin{aligned}
\operatorname{VAL}_{\mathbf{q}}\left(\mathcal{D}_{\emptyset}^{C}, \mathcal{F}\right) & =\sum_{j} \int_{0}^{t_{j}} f_{j}(y) x_{j}(y) y d y \\
& \leq \sum_{j} \int_{0}^{t_{j}} f_{j}(y) x_{j}(y) \beta_{j} d y+\sum_{j} \int_{0}^{t_{j}} f_{j}(y) x_{j}(y)\left(y-\beta_{j}\right) d y \\
& \leq \beta \cdot \mathbf{q}+\operatorname{VAL}\left(\mathcal{D}_{\emptyset}^{C}-\beta, \mathcal{F}\right) .
\end{aligned}
$$

We can recover $\operatorname{VAL}\left(\mathcal{D}_{\emptyset}^{C}-\beta, \mathcal{F}\right)$ using a two-part tariff for the original distribution $\mathcal{D}$ by employing the following concentration result proved by Rubinstein and Weinberg [2015], based on a result of Schechtman [1999].

LEMMA 5.4 (RUBINSTEIN AND WEINBERG [2015]). Let $\mathbf{v}$ be a constrained additive value function with a downwards closed feasibility constraint, drawn from a distribution over support $(-\infty, \tau]$ for some $\tau \geq 0$. Let a be the median of the value of the grand bundle, $\mathbf{v}([m])$. Then, $\mathbf{E}[\mathbf{v}([m])] \leq 3 a+4 \tau / \ln 2$.

LEMMA 5.5 .

$$
\operatorname{VAL}\left(\mathcal{D}_{\emptyset}^{C}-\beta, \mathcal{F}\right) \leq 6 \operatorname{BREV}(\mathcal{D}-\beta, \mathcal{F})+\frac{8}{\ln 2} \operatorname{SREV}_{\mathbf{q}}(\mathcal{D}, \mathcal{F})
$$

Proof. We apply Lemma 5.4 to the distribution $\mathcal{D}_{\emptyset}^{C}-\beta$ to obtain $\operatorname{VAL}\left(\mathcal{D}_{\emptyset}^{C}-\beta, \mathcal{F}\right) \leq$ $3 a+4 \tau / \ln 2$ where $a$ is the median of the value of the grand bundle under the distribution $\mathcal{D}_{\emptyset}^{C}-\beta$, and $\tau$ is the constant defined earlier.

Consider offering the grand bundle at price $a$ to a buyer with value drawn from $\mathcal{D}_{\emptyset}^{C}-\beta$; the buyer accepts with probability $1 / 2$. Therefore $\operatorname{BREV}(\mathcal{D}-\beta) \geq \operatorname{BREV}\left(\mathcal{D}_{\emptyset}^{C}-\right.$ $\beta) \geq a / 2$. Next, suppose that $\tau>0$. Consider selling the items separately at prices $t_{j}$ for all $j$. Recall that $\tau>0$ implies that $\mathbf{P r}_{\mathbf{v} \sim \mathcal{D}}\left[\exists j\right.$ s.t. $\left.v_{j}>t_{j}\right]=1-\rho_{\emptyset}=1 / 2$. So the agent buys at least one item with probability $1 / 2$. Noting that $t_{j}>\tau$ for all $j$, this item pricing obtains a revenue of at least $\tau / 2$. Since also $t_{j} \geq \beta_{j}$ for all $j$, we have $\tau \leq 2 \operatorname{SREV}_{\mathbf{q}}(\mathcal{D}, \mathcal{F})$.

\subsection{Putting the Pieces Together}

Combining Lemmas 5.1, 5.2, 5.3, and 5.5 together, we obtain the main result of this section:

LEMMA 5.6. For any product value distribution $\mathcal{D}$, downward closed feasibility constraint $\mathcal{F}$ and ex ante constraints $\mathbf{q}$,

$$
\operatorname{REV}_{\mathbf{q}}(\mathcal{D}, \mathcal{F}) \leq 6 \operatorname{BREV}(\mathcal{D}-\beta, \mathcal{F})+8(1+\ln 2+1 / \ln 2) \operatorname{SREV}_{\mathbf{q}}(\mathcal{D}, \mathcal{F})+\beta \cdot \mathbf{q} .
$$

It remains to bound the $\beta \cdot \mathrm{q}$ term. Note that this term is the revenue that would be obtained in the absence of any demand constraint (equivalently, in the additive setting) by setting the ex ante prices on the items. When $\mathcal{F}$ is a partition matroid and if the ex ante constraint q lies in the shrunk polytope $\frac{1}{2} \mathcal{P}_{\mathcal{F}}$, Chawla et al. [2010a] show via a prophet inequality that the term $\beta \cdot \mathbf{q}$ is bounded by the revenue of an item pricing.

Lemma 5.7 (ChaWla ET AL. [2010A]). For a partition matroid $\mathcal{F}$, ex ante constraints $\mathbf{q} \in \frac{1}{2} \mathcal{P}_{\mathcal{F}}$, and corresponding ex ante prices $\beta$,

$$
\beta \cdot \mathbf{q} \leq 2 \operatorname{SREV}_{\mathbf{q}}(\mathcal{D}, \mathcal{F}) .
$$


No prophet inequality based on static thresholds is known for general matroids. However, Feldman et al. [2016] nonetheless show that, if $q \in b \mathcal{P}_{\mathcal{F}}$, selling at the ex ante prices recovers a $(1-b)$ fraction of the relaxed revenue under a stronger demand constraint. This leads to the following result.

LEMMA 5.8 (FELDMAN ET AL. [2016]). For a general matroid $\mathcal{F}$, constant $b \in$ $(0,1)$, ex ante constraints $\mathbf{q} \in b \mathcal{P}_{\mathcal{F}}$, and corresponding ex ante prices $\beta$, there exists a submatroid $\mathcal{F}^{\prime} \subseteq \mathcal{F}$ such that

$$
\beta \cdot \mathbf{q} \leq \frac{1}{1-b} \operatorname{SREV}_{\mathbf{q}}\left(\mathcal{D}, \mathcal{F}^{\prime}\right) .
$$

Furthermore, the constraint $\mathcal{F}^{\prime}$ is efficiently computable.

We are now ready to prove Lemma 3.4 and Corollary 3.7, stated in Section 3.

LEMMA 3.4. Let $\mathcal{D}$ be any product value distribution and $\mathcal{F}$ be a matroid with feasible polytope $\mathcal{P}_{\mathcal{F}}$. Then, for any $q \in \frac{1}{2} \mathcal{P}_{\mathcal{F}}$, there exists a submatroid $\mathcal{F}^{\prime} \subseteq \mathcal{F}$ such that

$$
\operatorname{REV}_{\mathbf{q}}(\mathcal{D}, \mathcal{F}) \leq 33.1 \operatorname{TREV}_{\mathbf{q}}\left(\mathcal{D}, \mathcal{F}^{\prime}\right)
$$

If $\mathcal{F}$ is a partition matroid, then $\mathcal{F}^{\prime}=\mathcal{F}$.

Proof. We first observe that $\operatorname{BREV}(\mathcal{D}-\beta, \mathcal{F}) \leq \operatorname{TREV}_{\mathbf{q}}(\mathcal{D}, \mathcal{F})$. In particular, for any $a>0$, a two-part tariff with entry fee $a$ and item prices $\beta$ achieves at least as much revenue over values drawn from $\mathcal{D}$ as does a bundle pricing with price $a$ over values drawn from $\mathcal{D}-\beta$. The lemma now follows from Lemma 5.6, together with the bounds on $\beta \cdot \mathbf{q}$ given by Lemmas 5.7 and 5.8

As a final remark, we note that the condition $q \in \frac{1}{2} \mathcal{P}_{\mathcal{F}}$ in Lemma 3.4 is necessary only to recover $\beta \cdot \mathbf{q}$; we can, in fact, show a slightly weaker result which holds for arbitrary q.

COROLlaRY 3.7. Let $\mathcal{D}$ be any product value distribution and $\mathcal{F}$ be a matroid. Then for any $q \in[0,1]^{m}$, there exists a submatroid $\mathcal{F}^{\prime} \subseteq \mathcal{F}$ such that

$$
\operatorname{REV}_{\mathbf{q}}(\mathcal{D}, \mathcal{F}) \leq 35.1 \operatorname{TREV}_{\mathbf{q}}\left(\mathcal{D}, \mathcal{F}^{\prime}\right)
$$

If $\mathcal{F}$ is a partition matroid, then $\mathcal{F}^{\prime}=\mathcal{F}$.

PRoof. For any $q \in[0,1]^{m}$,

$$
\operatorname{REV}_{\mathbf{q}}(\mathcal{D}, \mathcal{F}) \leq \max _{\substack{\mathbf{q}^{\prime} \leq \mathbf{q} \\ \mathbf{q}^{\prime} \in \mathcal{P}_{\mathcal{F}}}} \operatorname{REV}_{\mathbf{q}^{\prime}}(\mathcal{D}, \mathcal{F})
$$

Therefore, there exists $\mathrm{q}^{\prime} \in \mathcal{P}_{\mathcal{F}}$ and corresponding $\beta^{\prime}$, such that Lemma 5.6 gives $\operatorname{REV}_{\mathbf{q}}(\mathcal{D}, \mathcal{F}) \leq 31.1 \operatorname{TREV}_{\mathbf{q}^{\prime}}(\mathcal{D}, \mathcal{F})+\beta^{\prime} \cdot \mathbf{q}^{\prime}$. Furthermore, by scaling $\mathbf{q}^{\prime}$ to lie in $\frac{1}{2} \mathcal{P}_{\mathcal{F}}$ we can only increase the corresponding ex ante prices, so Lemma 5.8 gives $\beta^{\prime} \cdot \mathbf{q}^{\prime} \leq$ $4 \operatorname{SREV}_{\mathbf{q}^{\prime}}\left(\mathcal{D}, \mathcal{F}^{\prime}\right)$ for some $\mathcal{F}^{\prime} \subseteq \mathcal{F}$. The corollary now follows by noting $\operatorname{TREV}_{\mathbf{q}^{\prime}}(\mathcal{D}, \mathcal{F}) \leq$ $\operatorname{TREv}_{\mathbf{q}}(\mathcal{D}, \mathcal{F})$ and $\operatorname{SREv}_{\mathbf{q}^{\prime}}\left(\mathcal{D}, \mathcal{F}^{\prime}\right) \leq \operatorname{SREv}_{\mathbf{q}}\left(\mathcal{D}, \mathcal{F}^{\prime}\right)$.

\subsection{Core decomposition with ex ante constraints}

The proof of Lemma 5.1 makes use of the following two lemmas, which are analogous to results proved by Babaioff et al. after $\mathrm{Li}$ and Yao.

Lemma 5.9. There exists a set $\left\{\mathbf{q}^{A} \in[0,1]^{m}: A \subseteq[m]\right\}$ such that $\sum_{A} \rho_{A} q_{j}^{A} \leq q_{j}$ for all $j$ and

$$
\operatorname{REV}_{\mathbf{q}}(\mathcal{D}, \mathcal{F}) \leq \sum_{A \subseteq[m]} \rho_{A} \operatorname{REv}_{\mathbf{q}^{A}}\left(\mathcal{D}_{A}, \mathcal{F}\right)
$$


Proof. Let $\mathcal{M}$ be a BIC mechanism which is q-constrained under $\mathcal{D}$ such that $\operatorname{REV}^{\mathcal{M}}(\mathcal{D})=\operatorname{REV}_{\mathbf{q}}(\mathcal{D}, \mathcal{F})$. So $\operatorname{REV}^{\mathcal{M}}(\mathcal{D})=\sum_{A \subseteq[m]} \rho_{A} \operatorname{REV}^{\mathcal{M}}\left(\mathcal{D}_{A}\right)$. Let $q_{j}^{A}$ be the probability that $\mathcal{M}$ allocates item $j$ given that $\mathbf{v}$ is drawn from $\mathcal{D}_{A}$; that is, $q_{j}^{A}=$ $\mathbf{E}_{\mathbf{v} \sim \mathcal{D}_{A}}\left[x_{j}(\mathbf{v})\right]$. Clearly $\sum_{A} \rho_{A} q_{j}^{A} \leq q_{j}$, by the assumption that $\mathcal{M}$ is q-constrained. The result follows since $\operatorname{REV}^{\mathcal{M}}\left(\mathcal{D}_{A}\right) \leq \operatorname{REV}_{\mathbf{q}^{A}}\left(\mathcal{D}_{A}, \mathcal{F}\right)$ for each $A$.

LEMMA 5.10. For any two independent distributions $\mathcal{D}_{S}$ and $\mathcal{D}_{T}$ over disjoint sets of items $S$ and $T$ with corresponding ex ante constraints $\mathbf{q}_{S}$ and $\mathbf{q}_{T}$ and a joint feasibility constraint $\mathcal{F}$,

$$
\operatorname{REV}_{\left(\mathbf{q}_{S} ; \mathbf{q}_{T}\right)}\left(\mathcal{D}_{S} \times \mathcal{D}_{T}, \mathcal{F}\right) \leq \operatorname{VAL}_{\mathbf{q}_{S}}\left(\mathcal{D}_{S},\left.\mathcal{F}\right|_{S}\right)+\operatorname{REV}_{\mathbf{q}_{T}}\left(\mathcal{D}_{T},\left.\mathcal{F}\right|_{T}\right) .
$$

Proof. Let $\mathcal{M}$ be a BIC mechanism which is $\left(\mathbf{q}_{S} ; \mathbf{q}_{T}\right)$-constrained under $\left(\mathcal{D}_{S} \times \mathcal{D}_{T}\right)$ such that $\operatorname{REV}^{\mathcal{M}}\left(\mathcal{D}_{S} \times \mathcal{D}_{T}\right)=\operatorname{REV}_{\left(\mathbf{q}_{S} ; \mathbf{q}_{T}\right)}\left(\mathcal{D}_{S} \times \mathcal{D}_{T}, \mathcal{F}\right)$. We construct a mechanism $\mathcal{M}^{\prime}$ for selling items in $T$ as follows. $\mathcal{M}^{\prime}$ first samples $\mathbf{v}_{S} \sim \mathcal{D}_{S}$, and then solicits a bid $\mathbf{v}_{T}$ for items in $T$. Let $\left(\mathbf{x}_{S \cup T}\left(\mathbf{v}_{S} ; \mathbf{v}_{T}\right), p\left(\mathbf{v}_{S} ; \mathbf{v}_{T}\right)\right)$ be the allocation returned and payment charged by $\mathcal{M}$ for the combined bid; then $\mathcal{M}^{\prime}$ returns the allocation $\mathbf{x}_{T}\left(\mathbf{v}_{S} ; \mathbf{v}_{T}\right)$ and charges $p\left(\mathbf{v}_{S} ; \mathbf{v}_{T}\right)-\mathbf{v}_{S}\left(\mathbf{x}_{S}\left(\mathbf{v}_{S} ; \mathbf{v}_{T}\right)\right)$.

We now prove that $\mathcal{M}^{\prime}$ is truthful. Suppose the bidder submits a bid $\mathbf{v}_{T}^{\prime}$. His utility is $\mathbf{v}_{T}\left(\mathbf{x}_{T}\left(\mathbf{v}_{S} ; \mathbf{v}_{T}^{\prime}\right)\right)-\left(p\left(\mathbf{v}_{S} ; \mathbf{v}_{T}^{\prime}\right)-\mathbf{v}_{S}\left(\mathbf{x}_{S}\left(\mathbf{v}_{S} ; \mathbf{v}_{T}^{\prime}\right)\right)\right)$, which is the utility of a bidder participating in $\mathcal{M}$ with valuation $\left(\mathbf{v}_{S}, \mathbf{v}_{T}^{\prime}\right)$. Since $\mathcal{M}$ is truthful, the bidder can do no worse by bidding $\mathbf{v}_{T}$ in $\mathcal{M}^{\prime}$ and receiving the utility of an agent who bids truthfully in $\mathcal{M}$.

Note that $\mathcal{M}^{\prime}$ allocates item $j \in T$ exactly when $\mathcal{M}$ does (conditioned on $\mathbf{v}_{S}$ ). So $\mathcal{M}^{\prime}$ is demand-feasible. Furthermore, since $\mathcal{M}^{\prime}$ draws $\mathbf{v}_{S}$ from $\mathcal{D}_{S}, \mathcal{M}^{\prime}$ is also $\mathbf{q}_{T}$-constrained under $\mathcal{D}_{T}$. Formally, let $\mathbf{x}^{\prime}$ be the allocation rule of $\mathcal{M}^{\prime}$; then $\mathbf{E}_{\mathbf{v}_{T} \sim \mathcal{D}_{T}}\left[x_{j}{ }^{\prime}\left(\mathbf{v}_{T}\right)\right]=$ $\mathbf{E}_{\mathbf{v}_{S} \sim \mathcal{D}_{S}, \mathbf{v}_{T} \sim \mathcal{D}_{T}}\left[x_{j}\left(\mathbf{v}_{S} ; \mathbf{v}_{T}\right)\right] \leq q_{j}$ for all $j \in T$.

The revenue obtained by $\mathcal{M}^{\prime}$ is

$$
\begin{aligned}
\operatorname{REV}^{\mathcal{M}^{\prime}}\left(\mathcal{D}_{T}\right) & =\mathbf{E}_{\mathbf{v}_{S} \sim \mathcal{D}_{S}, \mathbf{v}_{T} \sim \mathcal{D}_{T}}\left[p\left(\mathbf{v}_{S} ; \mathbf{v}_{T}\right)-\mathbf{v}_{S}\left(\mathbf{x}_{S}\left(\mathbf{v}_{S} ; \mathbf{v}_{T}\right)\right)\right] \\
& =\operatorname{REV}_{\left(\mathbf{q}_{S} ; \mathbf{q}_{T}\right)}\left(\mathcal{D}_{S} \times \mathcal{D}_{T}, \mathcal{F}\right)-\mathbf{E}_{\mathbf{v}_{S} \sim \mathcal{D}_{S}, \mathbf{v}_{T} \sim \mathcal{D}_{T}}\left[\mathbf{v}_{S}\left(\mathbf{x}_{S}\left(\mathbf{v}_{S} ; \mathbf{v}_{T}\right)\right)\right] \\
& \geq \operatorname{REV}_{\left(\mathbf{q}_{S} ; \mathbf{q}_{T}\right)}\left(\mathcal{D}_{S} \times \mathcal{D}_{T}, \mathcal{F}\right)-\operatorname{VAL}_{\mathbf{q}_{S}}\left(\mathcal{D}_{S},\left.\mathcal{F}\right|_{S}\right)
\end{aligned}
$$

where the inequality follows because the welfare $\mathcal{M}$ obtains from items in $S$ is a lower bound on the welfare of any $\mathbf{q}_{S}$-constrained mechanism for $\mathcal{D}_{S}$.

Proof Of Lemma 5.1. By Lemmas 5.9 and 5.10, we have

$$
\operatorname{REV}_{\mathbf{q}}(\mathcal{D}, \mathcal{F}) \leq \sum_{A \subseteq[m]} \rho_{A}\left(\operatorname{VAL}_{\mathbf{q}^{A}}\left(\mathcal{D}_{A}^{C},\left.\mathcal{F}\right|_{A^{c}}\right)+\operatorname{REV}_{\mathbf{q}^{A}}\left(\mathcal{D}_{A}^{T},\left.\mathcal{F}\right|_{A}\right)\right)
$$

For each $A \subseteq[m]$, let $\mathcal{M}^{A}$ be a truthful $\mathbf{q}^{A}$-constrained demand-feasible mechanism which obtains welfare equal to $\operatorname{VAL}_{\mathbf{q}^{A}}\left(\mathcal{D}_{A}^{C},\left.\mathcal{F}\right|_{A^{c}}\right)$. One way to allocate items when values are drawn from $\mathcal{D}_{\emptyset}^{C}$ is to choose to sell only items from some set $A \subseteq[m]$. Consider a mechanism which chooses from among all subsets of items, choosing $A$ with probability $\rho_{A}$, and then runs $\mathcal{M}^{A}$. The expected welfare from such a mechanism is exactly $\sum_{A \subseteq[m]} \rho_{A} \operatorname{VAL}_{\mathbf{q}^{A}}\left(\mathcal{D}_{A}^{C},\left.\mathcal{F}\right|_{A^{c}}\right)$. Since $\sum_{A \subseteq[m]} \rho_{A} q_{j}^{A} \leq q_{j}$, the welfare of this mechanism also provides a lower bound on $\operatorname{VAL}_{\mathbf{q}}\left(\mathcal{D}_{\emptyset}^{C}, \mathcal{F}\right)$.

\section{ACKNOWLEDGMENTS}

We are grateful to Anna Karlin for feedback on early drafts of this work, and to Jason Hartline for insights on efficiently solving the ex ante relaxation for symmetric agents.

This work is supported by the National Science Foundation, grant CCF-1101429 and grant CCF-1320854. 


\section{REFERENCES}

Saeed Alaei. 2011. Bayesian Combinatorial Auctions: Expanding Single Buyer Mechanisms to Many Buyers. In IEEE Symp. on Foundations of Computer Science. 512521.

Mark Armstrong. 1999. Price Discrimination by a Many-Product Firm. The Review of Economic Studies 66, 1 (1999), 151-168.

Moshe Babaioff, Nicole Immorlica, Brendan. Lucier, and S.Matthew Weinberg. 2014. A Simple and Approximately Optimal Mechanism for an Additive Buyer. In Foundations of Computer Science (FOCS), 2014 IEEE 55th Annual Symposium on. 21-30.

Patrick Briest, Shuchi Chawla, Robert Kleinberg, and S. Matthew Weinberg. 2010. Pricing Randomized Allocations. In ACM Symp. on Discrete Algorithms. 585-597.

Yang Cai, Constantinos Daskalakis, and S. Matthew Weinberg. 2012a. An algorithmic characterization of multi-dimensional mechanisms. In ACM Symp. on Theory of Computing. 459-478.

Yang Cai, Constantinos Daskalakis, and S. Matthew Weinberg. 2012b. Optimal Multidimensional Mechanism Design: Reducing Revenue to Welfare Maximization. In IEEE Symp. on Foundations of Computer Science. 130-139.

Yang Cai, Constantinos Daskalakis, and S. Matthew Weinberg. 2013a. Reducing Revenue to Welfare Maximization: Approximation Algorithms and other Generalizations. In ACM Symp. on Discrete Algorithms. 578-595.

Yang Cai, Constantinos Daskalakis, and S. Matthew Weinberg. 2013b. Understanding Incentives: Mechanism Design Becomes Algorithm Design. In IEEE Symp. on Foundations of Computer Science. 618-627.

Yang Cai, Nikhil Devanur, and S. Matthew Weinberg. 2016. A Duality Based Unified Approach to Bayesian Mechanism Design. In Proceedings of the 48th Annual ACM Symposium on Theory of Computing (STOC). To appear.

Shuchi Chawla, Jason Hartline, and Robert Kleinberg. 2007. Algorithmic Pricing via Virtual Valuations. In Proc. 9th ACM Conf. on Electronic Commerce. 243-251.

Shuchi Chawla, Jason D. Hartline, David L. Malec, and Balasubramanian Sivan. 2010a. Multi-parameter mechanism design and sequential posted pricing. In ACM Symp. on Theory of Computing. 311-320.

Shuchi Chawla, David Malec, and Balasubramanian Sivan. 2010b. The power of randomness in Bayesian optimal mechanism design. In Proc. 12th ACM Conf. on Electronic Commerce.

Shuchi Chawla and J. Benjamin Miller. 2016. Mechanism design for subadditive agents via an ex ante relaxation (full version). CoRR abs/1603.03806 (2016). http://arxiv.org/abs/1603.03806

Constantinos Daskalakis, Alan Deckelbaum, and Christos Tzamos. 2012. Optimal Pricing Is Hard. In Workshop on Internet and Network Economics (WINE). 298-308.

Constantinos Daskalakis, Alan Deckelbaum, and Christos Tzamos. 2014. The Complexity of Optimal Mechanism Design. In ACM Symp. on Discrete Algorithms. 13021318.

Constantinos Daskalakis, Alan Deckelbaum, and Christos Tzamos. 2015. Strong Duality for a Multiple-Good Monopolist. In Proceedings of the Sixteenth ACM Conference on Economics and Computation, EC '15. 449-450.

Uriel Feige, Vahab S. Mirrokni, and Jan Vondrák. 2011. Maximizing Non-monotone Submodular Functions. SIAM J. Comput. 40, 4 (July 2011), 1133-1153.

Moran Feldman, Ola Svensson, and Rico Zenklusen. 2016. Online Contention Resolution Schemes. In Proceedings of the Twenty-Seventh Annual ACM-SIAM Symposium on Discrete Algorithms (SODA '16). 1014-1033.

Yiannis Giannakopoulos and Elias Koutsoupias. 2014. Duality and Optimality of Auc- 
tions for Uniform Distributions. In Proceedings of the Fifteenth ACM Conference on Economics and Computation (EC '14). 259-276.

Yiannis Giannakopoulos and Elias Koutsoupias. 2015. Selling Two Goods Optimally. In Automata, Languages, and Programming: 42nd International Colloquium, ICALP 2015. 650-662.

Nima Haghpanah and Jason Hartline. 2015. Reverse Mechanism Design. In Proceedings of the Sixteenth ACM Conference on Economics and Computation (EC '15). 757 758.

Sergiu Hart and Noam Nisan. 2012. Approximate revenue maximization with multiple items. In ACM Conf. on Electronic Commerce. 656.

Sergiu Hart and Noam Nisan. 2013. The menu-size complexity of auctions. In ACM Conf. on Electronic Commerce. 565-566.

Jason Hartline. 2016. Mechanism Design and Approximation. http://jasonhartline. $\operatorname{com} / \mathrm{MDnA} /$

Robert Kleinberg and S. Matthew Weinberg. 2012. Matroid prophet inequalities. In ACM Symp. on Theory of Computing. 123-136.

Xinye Li and Andrew Chi-Chih Yao. 2013. On revenue maximization for selling multiple independently distributed items. Proceedings of the National Academy of Sciences (2013).

Philip J. Reny and Sergiu Hart. 2015. Maximal revenue with multiple goods: nonmonotonicity and other observations. Theoretical Economics 10, 3 (2015).

Aviad Rubinstein and S. Matthew Weinberg. 2015. Simple Mechanisms for a Subadditive Buyer and Applications to Revenue Monotonicity. In Proceedings of the Sixteenth ACM Conference on Economics and Computation, EC '15. 377-394.

Gideon Schechtman. 1999. Concentration, Results and Applications. (1999).

Andrew Chi-Chih Yao. 2015. An N-to-1 Bidder Reduction for Multi-item Auctions and Its Applications. In Proceedings of the Twenty-Sixth Annual ACM-SIAM Symposium on Discrete Algorithms (SODA '15). 92-109.

\section{A. MATROID CONCEPTS}

A matroid $M$ is a tuple $(G, \mathcal{I})$ where $G$ is called the ground set and $\mathcal{I} \subseteq 2^{G}$ is a collection of independent sets satisfying the following two properties:

(1) If $I \subseteq J$ and $J \in \mathcal{I}$, then $I \in \mathcal{I}$ ( $\mathcal{I}$ is downward-closed); and

(2) If $I, J \in \mathcal{I}$ and $|J|>|I|$, then there exists $e \in J \backslash I$ such that $(I \cup\{e\}) \in \mathcal{I}$.

A basis is an independent set of maximal size: $B \subseteq G$ is a basis if $B \in \mathcal{I}$ and $|I| \leq|B|$ for all $I \in \mathcal{I}$. The following lemma is a simple consequence of the fact that the greedy algorithm finds the maximum weight basis in any matroid.

LEMMA A.1. Let $\mathcal{F}$ be any matroid over ground set $G, I$ be any subset of $G$, and $w$ be any vector of weights defined on elements in $G$. If $j \in G$ belongs to a maximum weight basis of $\mathcal{F}$ and $j \in I$, then $j$ also belongs to a maximum weight basis of $\left.\mathcal{F}\right|_{I}$. 